\title{
Expandable intramedullary nailing and platelet rich plasma to treat long bone non-unions
}

\author{
Olimpio Galasso · Massimo Mariconda • \\ Gaetano Romano • Nicola Capuano · \\ Luigi Romano $\cdot$ Bruno Iannò $\cdot$ Carlo Milano
}

Received: 8 September 2007 / Accepted: 7 June 2008/Published online: 4 July 2008

(c) Springer-Verlag 2008

\begin{abstract}
Background Roentgenographic and functional outcomes of expandable self locking intramedullary nailing and platelet rich plasma (PRP) gel in the treatment of long bone non-unions are reported.

Materials and methods Twenty-two patients suffering from atrophic diaphyseal long bone non-unions were enrolled in the study. Patients were treated with removal of pre-existing hardware, decortication of non-union fragments, and fixation of pseudoarthrosis with expandable intramedullary nailing (Fixion ${ }^{\mathrm{TM}}$, Disc'O Tech, Tel Aviv, Israel). At surgery, PRP was placed in the pseudoarthrosis rim.

Results The thirteen-month follow-up showed 91\% (20/ 22 patients) of patients attaining bony union. The average

O. Galasso $(\bowtie) \cdot$ M. Mariconda · G. Romano · N. Capuano ·

L. Romano · B. Iannò · C. Milano

Cattedra ed Unità Operativa Complessa di Ortopedia e

Traumatologia, Università degli Studi "MagnaGræcia" di

Catanzaro, Campus "S. Venuta", V.le Europa, 88100 Germaneto

Catanzaro, Italy

e-mail: golimpiogo@hotmail.com

M. Mariconda · C. Milano

Dipartimento Assistenziale di Chirurgia, Ortopedia,

Traumatologia e Riabilitazione, Università degli Studi "Federico

II" di Napoli, Naples, Italy

G. Romano · L. Romano

Unità Operativa Complessa di Ortotraumatologia e Pronto

Soccorso Ortopedico Azienda Ospedaliera di rilievo nazionale

“A. Cardarelli", Naples, Italy

N. Capuano

Unità Operativa Complessa, Ospedale San Luca,

Vallo della Lucania, Salerno, Italy
\end{abstract}

time to union was 21.5 weeks. No infection, neurovascular complication, rotational malalignment, or limb shortening $>4 \mathrm{~mm}$ were observed. The healing rate of non-unions was comparable to that observed in previous studies but with a lower complication frequency.

Conclusions The combined use of self locking intramedullary nailing and PRP in the management of atrophic diaphyseal long bone non-unions seems to produce comparable results with less complications than previously reported. Further data are warranted to investigate the single contribution of PRP gel and Fixion nail.

Keywords Intramedullary nailing - Platelet rich plasma . Pseudoarthrosis

\section{Introduction}

Controversy on the treatment of long bone non-unions still exists among orthopaedic surgeons [1]. Primary goal in the treatment of these conditions is to achieve the healing of the fracture with satisfactory limb function. Internal fixation with lag screws and contoured plates to maintain or add compression has been proposed to manage bone nonunions, particularly in the upper limb [2]. The dynamic or static intramedullary nailing is the golden standard treatment for displaced non-unions of the lower limb [3].

Platelets play a major role in fracture healing. Indeed, the alpha granules of platelets, releasing several growth factors in the fracture rim, such as platelet-derived growth factor (PDGF), transforming growth factor beta (TGF- $\beta$ ), fibroblast growth factor (FGF- $\beta$ ), and vascular endothelial growth factor (VEGF) stimulate polymorphonuclear leukocytes, lymphocytes, monocytes, and macrophages [4]. TGF- $\beta$ and PDGF molecules show in vivo osteoinductive 
Fig. 1 a, b Non-union of the femur in a 32-year-old man treated with external fixation device 11 months before. The standard radiographs of the femur suggest cortical continuity
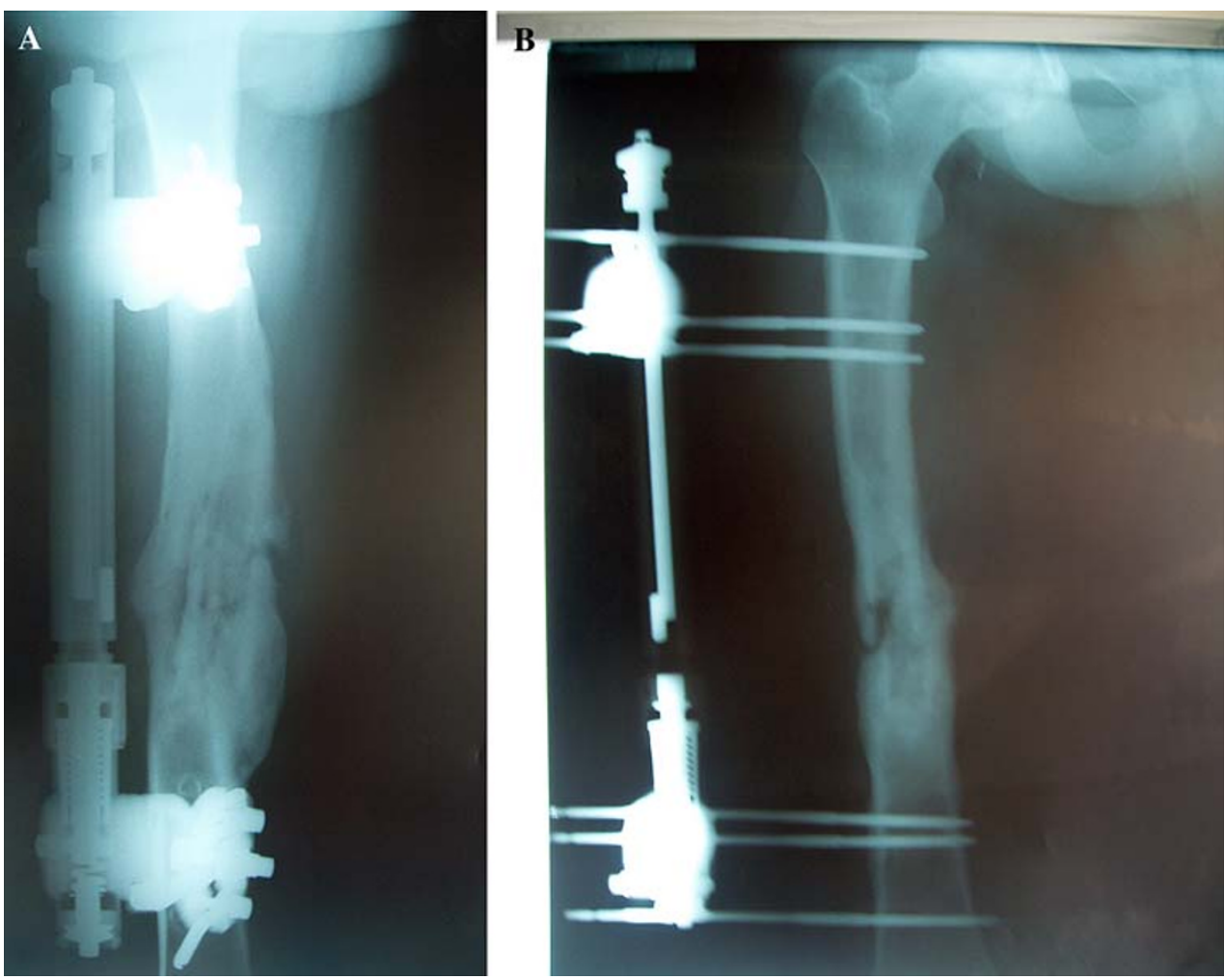

capacity [5, 6]. Moreover, VEGF enhances bone formation and bone healing by improving angiogenesis [7], and appears to be an appropriate tool to induce bone healing in atrophic non-unions [8]. Figs. 1, 2, 3, 4.

These issues suggested an autologous platelet concentrate can promote the bone-healing cascade [9].

The purpose of this study was to report the combined use of expandable intramedullary nailing and autologous platelet rich plasma (PRP) in the treatment of long bone non-unions.

\section{Materials and methods}

All patients with atrophic non-union of long bones admitted at our institutions in the years 2003-2004 who gave their informed consent were enrolled in the present study. This study was performed in accordance with the ethical standards of the 1964 Declaration of Helsinki and was approved by the Ethical Committee of our Institution. Non-union was defined as a fracture that failed to heal radiographically in 6 months [10]. Patients with age less than 18, infection, hypertrophic non-union, large bone defect in the site of nonunion, or preoperative platelet count of less than 100,000 cells $/ \mathrm{ml}$ were excluded. Twenty-two patients (13 males and 9 females), with a mean age of 39 years (range 20-56), fulfilled the inclusion criteria. At the time of the former fracture, 14 of 22 patients had been treated in a different
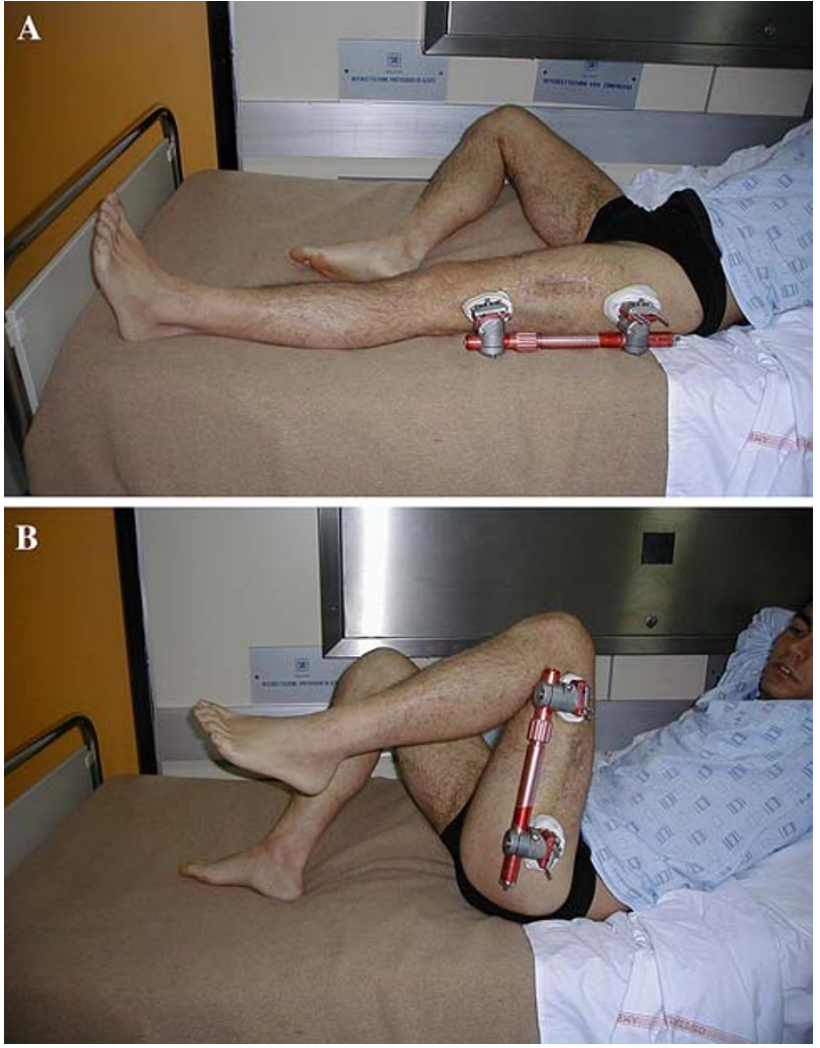

Fig. 2 a, b Clinical pictures carried out before surgery demonstrate full range of motion but the patient referred femoral pain at weight bearing 
Fig. 3 a, b, and $\mathbf{c}$ The CT scan with three-dimensional image shows obliteration of the medullary canal and absence of bony healing
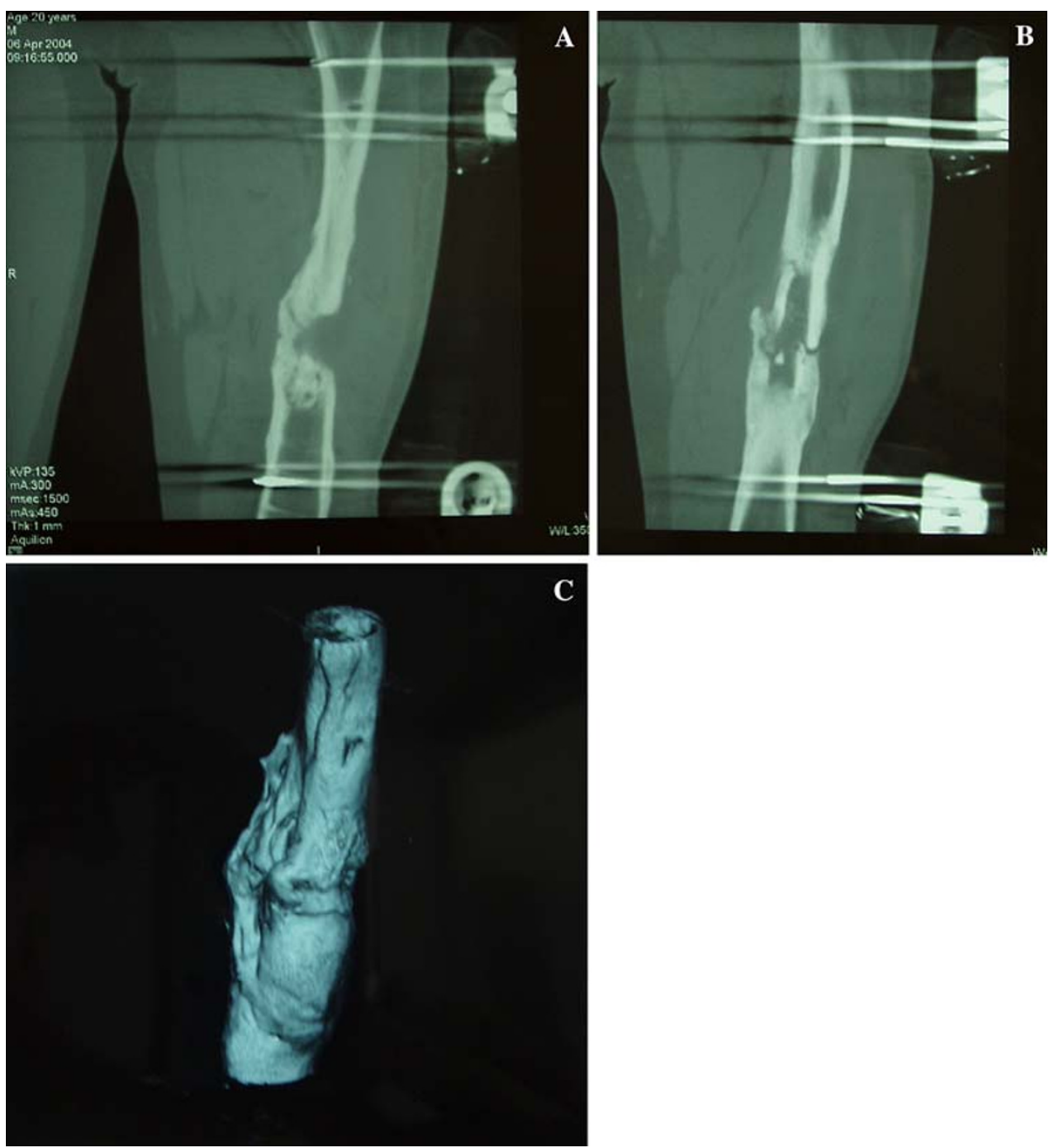

hospital. The site of non-union was the diaphysis of the following bones: tibia (11), femur (8), and humerus (3). The primary treatment of the fracture was conservative in two patients whereas external fixation and open reduction and internal fixation (ORIF) were the treatment originally performed in 11 and 9 patients, respectively. Five out of nine patients who had undergone ORIF received plate and screws fixation whereas the remnants had intramedullary nailing. After the informed consent was obtained, preoperative data were reported on a special form. The patient's clinical history, the educational level, the occupational status as well as the findings on physical examination were recorded. Early postoperative data were also obtained.

Short-term antibiotic and low-molecular weight heparin prophylaxis was used. The treatment, under peripheral anaesthesia, consisted of removal of previous metal implants (whenever present), decortication of the nonunion ends, reaming of the canal, and intramedullary nailing. Bacterial cultures were taken from the medullary canal. The Fixion nail was inserted by anterograde approach. It consists of a stainless steel core shaft and four perpendicular reinforcement bars, and is proximally fitted with a one-way valve. Its distal end is conically shaped. The nail, inserted in the medullary canal in its smallest diameter, is inflated using a manual disposable pump with high-pressurized saline solution. As the fluid enters the nail, the bars expand and a pressure gauge fixed to the pump measures the system pressure. The nail expands up to 70 bars, until it locks into the bone providing torsional stability and proper fragment alignment.

At the time of surgery, a platelet concentrate was obtained from a $55 \mathrm{cc}$. volume of the patient's blood by means of a centrifuge [11]. Six cc. of PRP was extracted from the buffy coat and separated from the platelet poor plasma. PRP was then activated with batroxobin and $\mathrm{CaCl}$ obtaining a gel [12] to be placed in the rim of non-union.

Patients treated for pseudoarthrosis of tibia or femur immediately started their postoperative rehabilitation 
Fig. 4 a, b, and $\mathbf{c}$ The X-ray examination carried out 4 months after nailing and application of PRP gel showed consolidation of pseudoarthrosis
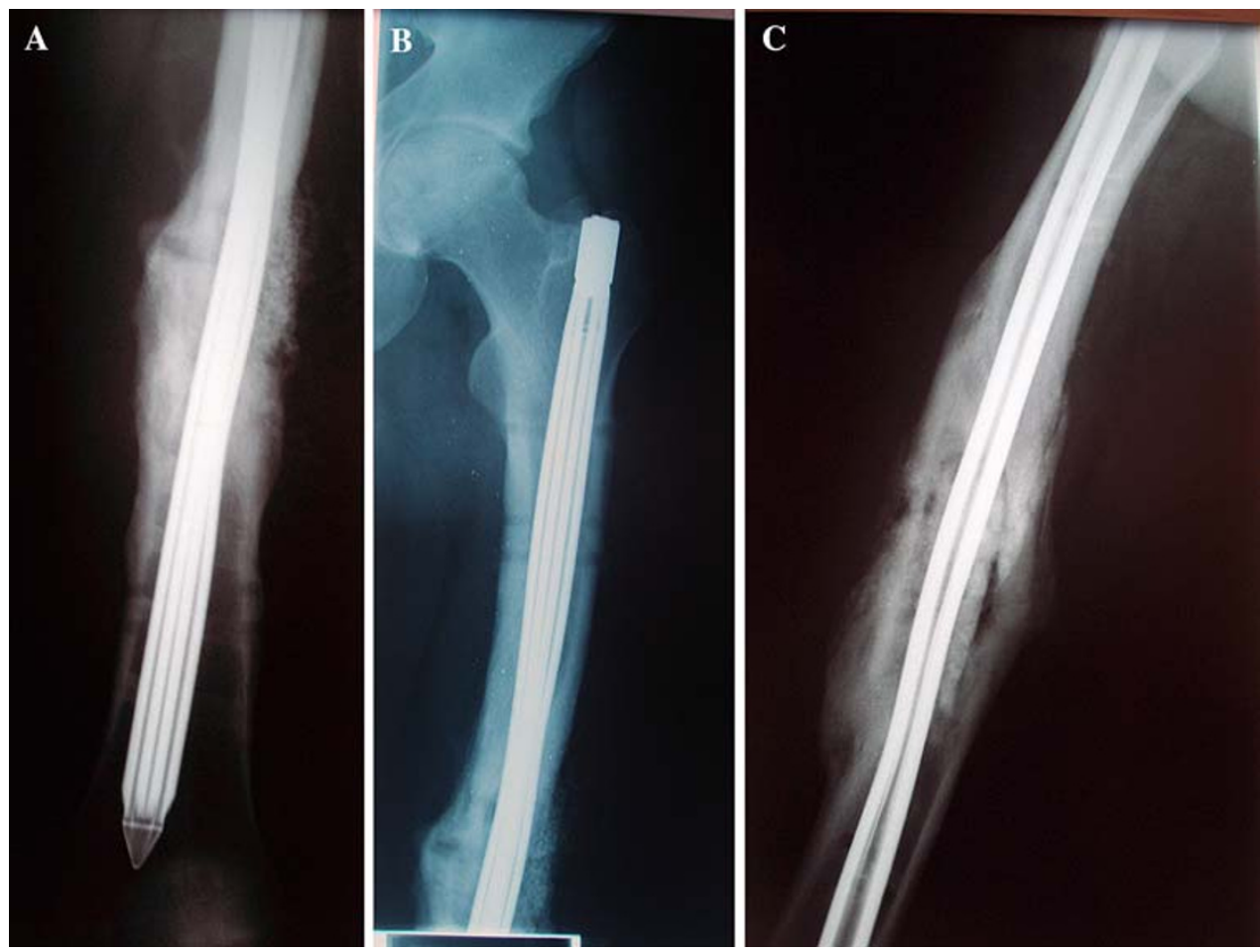

program and were allowed to partial weight bearing after 4 weeks. The patients with humeral non-union were immobilized in a plaster cast for 3 weeks before starting rehabilitation. Clinical and radiological evaluation was carried out every 45 days until the non-union healed. Final follow up was scheduled at 13 months. Union was defined when bone bridging was observed on the four cortices on the standard radiographic views. A computerized tomography scan was performed if required.

\section{Results}

The mean duration of surgery was 48 min (range 30-105) and the average fluoroscopy time was $2.6 \mathrm{~min}$ (range 1-5.5). Nail expansion was achieved in all surgeries but one: the tip of a nail was damaged during the insertion procedure in treatment of a femur pseudoarthrosis. The nail was replaced with a similar implant. No adverse effects such as damage to blood vessels, haematoma or delayed wound healing were noted using PRP. Temporary or permanent nerve damage that may result in pain or numbness did not occur and early or late postoperative infection were never observed.

No nail dislodgment or telescoping was noted throughout the treatment course. At final follow up 20 out of 22 patients $(91 \%)$ attained bony healing. Two non-unions, 1 femur and 1 tibia, failed to consolidate. The mean time to bony union was 21.5 weeks (range 12-26). Any rotational malalignment or limb shortening $>4 \mathrm{~mm}$ were not observed. Removal of nail was never required. As for the functional outcomes, 17 out of 19 patients originally suffering from pseudoarthrosis of the lower limb were able to walk without any support at final follow-up. On the followup control four patients reported that they had been forced to change their preoperative job, whereas 12 out of 22 were able to practice sport. A 20 degree of external motion limitation was noted in a patient treated for a non-union of the left humerus. This patient referred moderate pain and loss of abduction power.

\section{Discussion}

Intramedullary nailing is considered the gold standard in the treatment of long bone non-unions of the lower limb [3]. To the best of our knowledge, only one study reported results of Fixion nail in the treatment of long bone nonunions [13]. In the present paper combined treatment of long bone non-unions by expandable self locking nails and PRP gel is evaluated. The rate of healed non-unions and the mean time to union were similar to those reported in previous studies carried out with different nails and without PRP gel [14-16]. Kempf et al., using their nail, noted $92.6 \%$ and $94.8 \%$ of consolidation in femoral and tibial non-unions, respectively [17]. The average time to consolidation was shorter than that obtained in our series but a $7.5 \%$ infection rate and significant shortening of femur and 
tibia were reported [17]. The absence of interlocking screws with the related skin incisions and the short operative time could explain the lack of infection observed with expandable self locking nails. Moreover, the maintenance of limb length found in the present study could lie on the biomechanical characteristics of nail. Indeed, once the Fixion nail has expanded, it completely obliterates the medullary canal adapting to the canal's shape [18]. The nail fits into the isthmus of the shaft and therefore any axial and sideways displacement is prevented [19]. The large frictional contact surface between nail and bone results in a self-locking effect without the cortex weakening effect of drill holes. Forces acting on the outside of the bone are distributed over a large area and are not concentrated on small, localized areas, as when locking screws are used [20]. Mechanical testing of humeral Fixion nail showed an average strength in the 4-point-bending test of $43.15 \pm 3.0 \mathrm{Nm}$ and an average torsional stiffness of $2.78 \pm 0.27 \mathrm{Nm}^{2}$ [20], thus overcoming the results recorded for several conventional locking nails [20]. The lack of interlocking screws allowed short operative and fluoroscopy times; fluoroscopy was mainly used throughout the expansion phase, because of the concern to over expand the medullary canal. Daccarett et al., using the Fixion nail, obtained a $96 \%$ rate of healing with short time to consolidation $(13,15$, and 16 weeks treating pseudoarthrosis of femur, tibia, and humerus, respectively). However, a more heterogeneous group of patients was enrolled in this study consisting of both hypertrophic and atrophic non-unions [13].

In the present study the medullary canal was reamed up to two millimeters less than maximum diameter the nail reaches once its expansion is completed. In spite of several studies dealing with reaming procedure during intramedullary nailing of open or closed fracture [21-25], few data concerns reaming of medullary canal for pseudoarthrosis of bone. The choice of reaming the canal allowed the insertion of a nail with larger diameter, thus providing better stability. Moreover, the reaming produces an internal bone graft locally which appears to promote the healing of nonunions [26]. The cortical circulation and related periosteal callus formation have shown to be increased after reaming procedure [27]. The lack of a control group prevented us from comparing reaming and unreaming techniques. The absence of homogeneous group of patients treated with expandable intramedullary nailing alone did not allow to demonstrate the usefulness of PRP supplementation in the treatment of non-unions.

In the treatment of long bone non-unions, we obtained results similar to previous studies but with less complications using expandable intramedullary nails and PRP supplementation. Future controlled studies with larger sample size and more homogeneous study groups are warranted to investigate the single contribution of PRP gel and Fixion nail in the non-union treatment.

Conflict of interest statement Authors deny any commercial associations they have that might give rise to a conflict of interest in connection with the submitted article.

\section{References}

1. Rodriguez-Merchan EC (2004) Editorial comment. Clin Orthop Relat Res 419:1

2. Lemaire R (2000) Management of non-unions. An overview. In: Duparc J (ed) Surgical techniques in orthopaedics and traumatology. EFORT 1. Elsevier, Paris 55-030-F-10

3. Rosen H (1998) Non-union and Malunion. In: Browner BD, Jupiter JB, Levine AM, Trafton PG (eds) Skeletal Trauma, vol 1, 2nd edn. WB Saunders, Philadelphia, pp 619-660

4. Schmitt JM, Hwang K, Winn SR, Hollinger JO (1999) Bone morphogenetic proteins: An update on basic biology and clinical relevance. J Orthop Res 17:269-278

5. Lamerigts NM, Buma P, Aspenberg P, Schreurs BW, Slooff TJ (1999) Role of growth factors in the incorporation of unloaded bone allografts in the goat. Clin Orthop Relat Res 368:260-270

6. Bostrom MP, Asnis P (1998) Transforming growth factor beta in fracture repair. Clin Orthop Relat Res 355S:124-131

7. Peng H, Usas A, Olshanski A, Ho AM, Gearhart B, Cooper GM, Huard J (2005) VEGF improves, whereas sFlt1 inhibits, BMP2induced bone formation and bone healing through modulation of angiogenesis. J Bone Miner Res 20:2017-2027

8. Geiger F, Bertram H, Berger I, Lorenz H, Wall O, Eckhardt C, Simank HG, Richter W (2005) Vascular endothelial growth factor gene-activated matrix (VEGF165-GAM) enhances osteogenesis and angiogenesis in large segmental bone defects. J Bone Miner Res 20:2028-2035

9. Marx RE, Carlson ER, Eichstaedt RM, Schimmele SR, Strauss JE, Georgeff KR (1998) Platelet rich plasma: growth factor enhancement for bone grafts. Oral Surg 85:638-646

10. Bhandari M, Guyatt GH, Swiontkowski MF, Tornetta P, Sprague S, Schemitsch EH (2002) A lack of consensus in the assessment of fracture healing among orthopaedic surgeons. Orthop Trauma $16: 562-566$

11. Eppley BL, Woodell JE, Higgins J (2004) Platelet quantification and growth factor analysis from platelet-rich plasma: implications for wound healing. Plast Reconstr Surg 114:1502-1508

12. Gaffney PJ, Edgell TA (1998) The second British Standard for Batroxobin (moojeni). Tromb Haemost 80:1037-1038

13. Daccarett M, Walz BM, Seligson D (2004) Use of an expanadable nail in the treatment of non-union fractures of the femur, tibia, and humerus. Osteo Trauma Care 12:130-134

14. Ahlo A, Ekeland A, Stromsoe K (1993) Non-union of tibial shaft fractures treated with locked intramedullary nailing without bone grafting. J Trauma 34:62-67

15. Moed BR, Watson JT (1995) Intramedullary nailing of aseptic tibial non-unions without the use of the fracture table. J Orthop Trauma 9:128-134

16. Pihlajamaki HK, Salminen ST, Bostman OM (2002) The treatment of non-unions using intramedullary nailing of femoral shaft fractures. J Orthop Trauma 16:394-402

17. Kempf I, Grosse A, Rigault P (1986) The treatment of noninfected pseudoarthrosis of the femur and tibia with locked intramedullary nailing. Clin Orthop Relat Res 212:142-154

18. Blum J, Janzing H, Gahr R (2001) Clinical performance of a new medullar nail. J Orthopaed Traumatol 342-349 
19. Lepore S, Capuano N, Romano G (2000) Preliminary clinical and radiographic results with the Fixion Intramedullary Nail: an inflatable self-locking system for long bone fracture. J Orthopaed Traumatol 3:135-140

20. Franck WM, Olivieri M, Jannasch O, Hennig FF (2002) An expandable nailing system for the management of pathological humerus fractures. Arch Orthop Trauma Surg 122:400-405

21. Court-Brown CM, McQueen MM, Quaba AA, Christie J (1991) Locked intramedullary nailing of open tibial fractures. J Bone Joint Surg 73B:959-964

22. Heim D, Regazzoni P, Tsakiris DA, Aebi T, Schlegel U, Marbet GA, Perren SM (1995) Intramedullary nailing and pulmonary embolism: does unreamed nailing prevent embolization? An in vitro study in rabbit. J Trauma 38:899-906

23. Bonatus T, Olson SA, Lee S, Chapmann MW (1997) Non reamed locking intramedullary nailing for open fractures of the tibia. Clin Orthop Relat Res 339:58-64
24. Koval KJ, Clapper MF, Brumback RJ, Ellison PS Jr, Poka A, Bathon GH, Burgess AR (1991) Complications of reamed intramedullary nailing of the tibia. J Orthop Trauma 5:184-189

25. Klein MP, Rahn BA, Frigg R, Kessler S, Perren SM (1990) Reaming versus non-reaming in medullary nailing: Interference with cortical circulation of the canine tibia. Arch Ortop Trauma Surg 109:314-316

26. Schemitsch EH, Kowalski MJ, Swiontkowski MF, Senft D (1994) Cortical bone blood flow in reamed and unreamed locked intra medullary nailing: a fractured tibia model in sheep. J Orthop Trauma 8:373-382

27. Grundnes O, Reikeras O (1993) Acute effects of intramedullary reaming on bone blood flow in rats. Acta Orthop Scan 64:203206 